\title{
Correction to: A cluster randomized controlled trial for child and parent weight management: children and parents randomized to the intervention group have correlated changes in adiposity
}

Diane C. Berry ${ }^{1 *}$, Robert G. McMurray ${ }^{2}$, Todd A. Schwartz ${ }^{3}$, Emily G. Hall ${ }^{1}$, Madeline N. Neal ${ }^{1}$ and Reuben Adatorwovor ${ }^{3}$

\section{Erratum}

After publication of the original article [1] it was highlighted that the surname of author Reuben Adatorwovor was incorrectly typeset as Adatorwover. These errors were introduced during typesetting; thus the publisher apologizes for this error. Additionally, the original manuscript has also been updated to amend this error.

\section{Author details}

${ }^{1}$ School of Nursing, The University of North Carolina at Chapel Hill, Campus Box 7460, Chapel Hill, NC 27599-7460, USA. ${ }^{2}$ Department of Exercise and Sport Science, Department of Nutrition, The University of North Carolina at Chapel Hill, Campus Box 8700, Chapel Hill, NC 27599-8700, USA.

${ }^{3}$ Department of Biostatistics, Gillings School of Global Public Health and School of Nursing, The University of North Carolina at Chapel Hill, Campus Box 7420, Chapel Hill, NC 27599-7420, USA.

Published online: 26 January 2018

\section{Reference}

1. Berry DC, McMurray RG, Schwartz TA, Hall EG, Neal MN, Adatorwovor R. A cluster randomized controlled trial for child and parent weight management: children and parents randomized to the intervention group have correlated changes in adiposity. BMC Obes. 2017;4(1):39.

\footnotetext{
* Correspondence: dberry@email.unc.edu

${ }^{1}$ School of Nursing, The University of North Carolina at Chapel Hill, Campus Box 7460, Chapel Hill, NC 27599-7460, USA
} 